\title{
Neurobiology of developmental dyslexia: Part 1: A review of evidence from autopsy and structural neuro-imaging studies
}

\section{SO Wajuihian}

African Vision Research Institute (AVRI), University of KwaZulu-Natal, Private Bag X54001, Durban, 4000 South Africa

<swajuihian@mweb.co.za>

Received 9 May 2011; revised version accepted 25 October 2011

\begin{abstract}
Developmental dyslexia (DD) is a languagebased neurological disorder which impairs reading ability but does not result from low intelligence, lack of motivation, sensory impairment, or inadequate instruction. Although the neurological basis of dyslexia has long been assumed, the exact nature of the altered brain structure associated with DD remains unknown and has been a subject of autopsy and neuro-imaging research. Autopsy studies provide consistent evidence of symmetry of the planum temporale (PT), thalamus and cortical malformations, whereas results from structural imaging
\end{abstract}

studies such as computed tomography (CT) and magnetic resonance imaging (MRI) are inconsistent. To address the possible etiology of DD, this paper reviews evidence from autopsy and structural imaging studies on developmental dyslexia and discusses possible methodological sources of some inconsistent results. The role of the optometrist in the multidisciplinary management of dyslexia is highlighted. (S Afr Optom 2011 70(4) 191-202)

Key Words: Developmental dyslexia, autopsy, post-mortem, magnetic resonance imaging (MRI), optometrist.

\section{Introduction}

In 1968, the World Federation of Neurologists defined dyslexia as "a specific learning disability that is neurological in origin. It is characterized by difficulties with accurate and/or fluent word recognition and by poor spelling and decoding abilities. These difficulties typically result from a deficit in the phonological component of language that is often unexpected in relation to other cognitive abilities and the provision of effective classroom instruction. Secondary consequences may include problems in reading comprehension and reduced reading experience that can impede the growth of vocabulary and background knowledge" 1 .

From a historical perspective, converging reports on the neurobiology of developmental dyslexia reveals that earlier understandings of the neurobiology of development dyslexia was derived from studies by Morgan, Hinshelwood, Dejerine Wernicke and Bro$\mathrm{ca}^{2,3}$. Karl Wernicke, in 1874 (cited by Nakada) ${ }^{3}$, reported that a lesion in the left hemisphere resulted in a unique language disorder characterized by comprehension difficulties (Wernicke's aphasia). The lesions associated with Wernicke's aphasia are typically found on the superior surface of the temporal lobe between the auditory cortex and angular gyrus-the area of the cortex later known as the Wernicke's area ${ }^{2,3}$, the same area where a language-relevant structure, the planum temporale (PT) is located ${ }^{4}$. According to $\mathrm{Habib}^{2}$, it was first reported by Dejerine in 1891 that damage to the same region of the brain (angular gyrus) resulted 
in variable degree of impairments in reading and writing, which suggested that the left angular gyrus plays a role in reading impairments. Earliest descriptions of dyslexia therefore have related the disorder mainly to cerebral pathology.

Dyslexia is a language-based disorder 5,6 of neurobiological origin ${ }^{7,8}$ although the exact brain structure involved in the etiology of DD is still poorly understood. Consequently, studies investigating the neurobiological basis of dyslexia have focused on those neural systems subserving language, which are located in the Perisylvian association cortex in the left hemisphere of the brain ${ }^{9}, 10$. An approach to determining the neuroanatomical abnormality in a behavioural condition such as dyslexia is to study related microscopic (neuronal level) or macroscopic (molecular level) as structural abnormalities might correspond to the behavioural abnormalities characteristic of the disorder ${ }^{11}$. An understanding of the neurobiological basis of dyslexia will guide diagnosis and intervention ${ }^{7}$. Certainly, dyslexia is primarily not caused by vision anomalies but optometry plays an important role in addressing the vision needs of a dyslexic child, which then makes intervention easier. Consequently, despite the role of optometry in the multidisciplinary management of dyslexia, information on dyslexia is sparse in optometric literature. This paper therefore, serves to contribute to the understanding of dyslexia.

Using the available empirical evidence, studies of brain morphometry (measurement of brain structures) derived from postmortem and structural imaging studies are reviewed with the aim to address the question of whether anatomical deviations in patterns of brain asymmetry (a normal brain is asymmetrical) characterizes the brains of dyslexic persons. The focus of this review is on the PT (at the macroscopic level), cortical malformations (at the microscopic level) and the thalamus. The review is presented in three sections. The first section will review evidence from autopsy studies on the PT, cortical malformations and the thalamus and the second section will review studies using structural imaging studies on the PT. In the final section, the methodological limitations that contribute to inconsistent results are discussed. First, it is necessary to present a brief review of basic neuro-anatomy of language-relevant areas of the brain, which will enhance an understanding of subsequent papers on the neurobiology of DD.

\section{Basic neuro-anatomy of language-relevant struc- tures in the brain}

The brain, consisting of billions of neurons (nerve cells), which communicate with each other along an electrochemical path ${ }^{5,12-13}$ is divided into left and right hemispheres, connected by a bundle of nerve fibers, the corpus callosum. The cerebrum is the largest part of the brain and has a typical pattern of cellular arrangement (Cytoarchitecture) $)^{5,12-13}$. The part of the brain that is visible on the surface is called the cerebral cortex ${ }^{5}$ and is the layer of the brain often referred to as gray matter. The cortex covers the outer portion of the cerebrum and cerebellum 5 . The regions marked around the Sylvian fissure (which separates the temporal lobe from the frontal and parietal lobe) are called the perisylvian region ${ }^{5,12-13}$. The perisylvian region is on the surface of the brain (the cortex), and holds the majority of language tissue. The lower bank of the Sylvian fissure contains the primary auditory cortex on Heschl's gyrus and auditory association cortex on the planum ${ }^{5,12-13}$. Heschl's gyrus lies on the anterior boundary to the PT. The cerebral cortex has folds that allow the cortex to fit compactly into the skull. The folds of the cerebral cortex give the surface of the human brain its wrinkled appearance ("ridges and valleys") ${ }^{5,12-13}$. The ridges are called gyri and the valleys, sulci or fissures (small and large respectively). The largest sulci are sometimes called fissures. Several deep sulci divide the cortex into four areas: frontal, parietal, occipital, and temporal lobes ${ }^{5,12-13}$. The temporal lobe is a region of the cerebral cortex that is located beneath the Sylvian fissure on both cerebral hemispheres of the mammalian brain ${ }^{5,12-13}$. The auditory cortex, located in the temporal lobe is a highly organized processing unit of sound in the brain. The cortex area is the neural base of language in humans ${ }^{5,12-13}$. Another important anatomical structure is the angular gyrus-which is a part of the brain in the parietal lobe, that lies near the superior edge of the temporal lobe and is involved in a number of processes related to language, and cognition $5,12-13$. Language-related information comes from the auditory cortex (for spoken language) or from the visual cortex (for written language) ${ }^{3}$.

Also, relevant to the discussions of the neurobiology of dyslexia are the concept of lateralization, cerebral dominance and asymmetry. Brain lateralization is the phenomenon whereby a given function is 
preferentially controlled by one side of the brain relative to the other that is, two halves of the brain serve different functions. Cerebral dominance is an anatomical term that explains the superiority of one side of the brain for a particular function. Each half of the brain is dominant for several functions, for example, the left side is usually dominant for language, the right side for certain musical and spatial abilities. Brain asymmetry refers to a proportional difference between the right and left hemispheres of the brain ${ }^{9}$. As the PT is central to the subject of this review, a brief description of the PT is therefore warranted.

\section{The planum temporale (PT)}

Although there is controversy on how to define the exact anatomical borders, the PT has been conventionally described as a part of the temporal lobe, known to be relevant in language functions ${ }^{4,14-16}$. The PT is a roughly triangular structure, which lies on the superior surface of the temporal lobe (just adjacent to the first Heschl's gyrus) inside the Sylvian fissure (SF) and it is a region of the cortex that falls within the Wernicke's area, on the left hemisphere of the brain ${ }^{14-16}$.

According to Kusych et al17, although a relationship between the PT and language function was first inferred by Wernicke, research interest in the studies of brain morphometry of the PT were stimulated by the landmark study of Geschwind and Levitsky ${ }^{4}$. Gescwhind and Levisky ${ }^{4}$ performed autopsy examinations of the length of the PT in 100 normal brains and found it to be longer on the left in $65 \%$, symmetrical in $25 \%$, and shorter on the left in only $10 \%$ of the sample. The authors therefore hypothesized that the larger left PT might be an anatomical indicator for the specialization of the left hemisphere for language ${ }^{4}$. Since the PT lies within the classical posterior language region in the left hemisphere, defective development of language-related abilities in dyslexic subjects was thought to be related to a lack of asymmetry ${ }^{18-20}$. Individuals with unusual asymmetry (symmetrical) of the PT are at risk of being dyslexic because their left hemispheres are not as structurally adapted for language as they are in individuals with usual patterns of asymmetry ${ }^{20}$. The PT is also considered an anatomical structure in language because it serves as the foundation for the auditory association cortex (a posterior language area in the left hemisphere) and it is part of the classical Wernicke's area ${ }^{14,20}$. Also, the PT appears to have cytoarchitectonic properties which are necessary to relate the anatomical structure to functional significance 20,21 . Furthermore, since the PT asymmetry is apparent by the 29th to 31st week of gestation, abnormalities in this brain region may suggest a disruption of neurodevelopmental processes involved in establishing functional hemispheric lateralization ${ }^{16}$. Lastly, neuroimaging studies ${ }^{3,17,22}$ have shown that the PT plays an important role in language processing. Given the role of the planum temporale in language function and dyslexia being a languagebased disorder, histo-pathological and neuro-imaging studies have been focused on the PT. These studies are reviewed below.

\section{A review of the literature}

Studies of brain morphology tend to follow a chronological pattern. Studies conducted before the 1980s were mainly autopsy examinations, studies in the early 1990s were structural imaging studies (CTs and MRIs) while studies conducted from the late 1990s till date were mainly on functional imaging (conducted while an individual performs a specific task such as reading).

\section{(1) Autopsy Studies}

Autopsy studies investigate the brains of developmental dyslexics that have died from illness or accident. Anatomically, dyslexic brains have been found to be structurally different from those of non-dyslexic brains ${ }^{23-29}$. Evidence from autopsy studies revealed anomalies at two levels: microscopic (neuronal level) differences on cortex that includes ectopias, dysplesia and microgyria and macroscopic differences which include symmetry in the PT $23-24$.

The study by Drake ${ }^{30}$ in 1968 was the first autopsy study to reflect on the neuro-pathological basis of developmental dyslexia. Drake ${ }^{30}$ reported the case of a 12-year-old child, who had marked difficulties in reading comprehension, problems in arithmetic, poor spelling and recurring frontal headaches. Postmortem (autopsy) examination of the brain revealed a massive hemorrhage in the inferior vermis of the cerebellum, 
an abnormal convulated pattern bilaterally in the parietal lobes, a thinned corpus callosum, and ectopic neurons deep in the white matter. There was no report on the asymmetry of the PT.

\section{(i) Findings on the PT}

Galaburda and colleagues ${ }^{23-29}$ have been the main researchers in the area of autopsy studies of persons with documented histories of dyslexic brains and have studied several dyslexic brains until date. In 1979, Galaburda and Kemper ${ }^{21}$ reported the postmortem results of case of a 20 year old dyslexic man who had a family history of dyslexia. In 1985, Galaburda et $a^{23}$ reported three additional postmortem findings of three males, aged 14, 20 and 32 years respectively. Later in 1989, Humphrey et al 24 (working with Galaburda' et al) reported the neuro-patholological findings in three female dyslexic patients. So far, a total of 11 autopsied dyslexic brains (eight male, three females) have been studied by Galaburda and colleagues ${ }^{23-29}$. A remarkable finding with postmortem examination by Galaburda and colleagues is that all eight postmortem cases that examined the PT (specifically) of dyslexic subjects (six males and two females) had symmetrical PT $^{25}$ (normal brains have asymmetrical PT) ${ }^{4}$. Galaburda argued that because less than $33 \%$ of ordinary brains have symmetrical PT, the probability of encountering eight dyslexic brains with symmetrical PT by chance is minimal ${ }^{25}$. The reported symmetry in the dyslexic subjects resulted from a bilaterally large PT, (compared to those in normal brains) rather than from a reduction on the left $\mathrm{PT}^{31}$. In addition, this enlargement of the PT suggested anomalous brain development during the later stages of (prenatal) corticogenesis, which may result in abnormally high levels of surviving neurons with a subsequent restructuring of cortical architecture ${ }^{23}$. This, in effect, means that the mechanism for eliminating unwanted cells is defective in dyslexia forming a kind of "miswiring" in the brain" 23 .

\section{(ii) Cortical malformations}

In addition to the findings of symmetrical PT, Galburda et al 21,23-24 also observed that the dyslexics' cerebral cortexs contained some cortical malformations in the form of neuronal ectopias, architectonic dysplesias (focally distorted cortical architecture) ${ }^{32}$ and microgyria (abnormal infoldings) ${ }^{21,23,33}$ in eight of their male and one of the female dyslexic postmortem samples ${ }^{33}$. Ectopias are misplaced cells comprising brain cells and glia (supporting cells) that are located in areas of the cortex that should not have them. These misplaced cells are neurons located unusually in layer one of the cerebral cortex and are devoid of nerve cells ${ }^{23,25}$. Ectopias consist of 50-100 neurons (and glia) that during neural migration, have missed their target in the cortex and escaped into the molecular layer, through a breach in the external glial limiting membrane, accompanied by mild disorganization of the underlying cortical layers which cause a loss of characteristic architectural organization of the cortical neuron (microgyrias and dysplasia). The ectopias and the disordered cortical layering are together referred to as microdysgenesis ${ }^{25}, 34$. More so, the number and location of the focal cortical abnormalities vary from brain to brain, affect the language-relevant perisylvian cortex and tend to be more frequent on the left side of the brain than the right ${ }^{25}, 34$. Ectopias arise developmentally before the completion of the period of neuronal migration to the neocortex, which in humans takes place between 16 and 20 weeks of gestation ${ }^{34}$

Ectopias are occasionally seen in routine autopsy studies but only in less than 15 percent of the time, and tend to be few and usually not perisylvian in location $^{25}$. The type of neuronal migration disorder seen in the dyslexic brains may be present in other conditions such as fetal alcohol syndrome and nonspecific mental retardation but those in the latter conditions resulted from obvious injury from the brain during development ${ }^{25}, 34$. In the dyslexic brains, on the other hand, it was more subtle and restricted in location $^{25}$. In Galaburda's reports, ectopias were seen in all dyslexic male cases and in one out of two female cases ${ }^{25,34}$. Overall, the dyslexic female brains showed fewer and differently located cortical malformations ${ }^{24}$. These cortical anomalies contain neurons born at different times during histogenesis of the cortex. They lead to alterations in the pattern of connectivity within and between the hemispheres especially with the thalamus, ipsilateral cortex and contralateral cortex and affect the development of these brain areas $^{33,34}$.

An important perspective to the autopsy findings of Galaburda and colleagues ${ }^{24-25}$ is that both reduced PT asymmetry and aberrant cortical malformations 
represent significant neuroanatomical association to developmental dyslexia, even if the symmetry of the brain regions is not classified as pathological and both deviations resulted from abnormal neuronal migration $^{25}$. Neuronal migration refers to a period in brain development when young neurons migrate in search of their final locations in the brain. Neurons are born from neural stem cells in specific proliferative zones far away from the brain areas to their target destination. Neuronal migration, a process which takes place in the cerebral cortex of the brain occurs in an orderly pattern ${ }^{9,35-36}$ and is guided by specialized cells (radial glial cells) that occupy the developing cortex which ultimately gives rise to new neurons through cell division. The termination of migration therefore depends on the integrity of radial cells ${ }^{9,36}$. In some cases, neurons must travel through great distances to reach the cortex. Very tight controls (which are influenced by gene functions) must be in place for neurons to end up in the right place ${ }^{9,35-36}$. Loss of radial glial cell integrity can therefore cause aberrations in migration anywhere along the neuronal migration path ${ }^{9,36}$. Normal neuronal migration is also dependent on intracellular mechanisms within newly generated neuronal cells that allow for cellular motility. Disruptions in neuronal motility, in radial glia integrity, or in the adhesion between radial glial and new neurons can alter the normal developmental patterning and migration in the neocortex which contribute to establishing abnormal neuronal circuits in brain areas typically devoted to language function 9,36 .

\section{(iii) Findings in the thalamus}

Beside autopsy examinations on the PT, Galaburda and colleagues ${ }^{27-29}$ also performed post-mortem examinations on human dyslexics' thalamus. Specifically, the lateral geniculate nucleus (LGN) of the visual pathway ${ }^{29}$, the medial geniculate nucleus (MGN) of the auditory pathway ${ }^{28}$ and another study 27 investigated histological changes in the LGN of the primary visual cortex (Broadman area 17). In the LGN, Livingstone et $a^{29}$ found that the magnocellular layers of the visual pathway were more disorganized in dyslexic brains and the cell bodies appeared smaller. The decreased magnocellular geniculate neurons have functional consequences while the smaller cell bodies are likely to have thinner axons with slower conduction velocities ${ }^{29}$. The magnocellular system is particu- larly important for the control of eye movements and visual attention ${ }^{29}$. In the auditory system, Galaburda et al${ }^{28}$ reported significantly smaller MGN neurons on the left side compared with the right in the same dyslexic autopsy specimens. No hemispheric asymmetry in MGN neuronal size was observed in ordinary brains. In the primary visual cortex, dyslexic brains exhibited histologic changes in the magnocellular cells of LGN as well as abnormal visually evoked potentials and brain activation to magnospecific stimuli ${ }^{27}$. Overall, the structural deviations found in the LGN of dyslexic brains may be responsible for slowness in early segments of the magnocellular channels, whereas the MGN differences may relate to the auditory temporal processing abnormalities in dyslexia ${ }^{27-29}$.

\section{Demerits of autopsy examinations}

Although autopsy studies are important in providing direct neuropathological evidence of morphological abnormalities associated with DD and in providing a basis for neuroimaging studies, there are several limitations with the application of such findings. Such limitations include: (i) generalizability is limited due to small sample size (ii) with increased period of storage of autopsy specimens, there is a higher risk of cell shrinkage ${ }^{37}$ (iii) reliable identification of microanatomical deviances in general and the boundaries of the PT in particular are difficult to obtain $^{37-39}$ (iv) all the subjects' historical data was retrospective therefore; it is unclear to what extent brains studied are representative of dyslexic brains in general, (v) autopsy studies are expensive 15, 38-39 and specimens are scarce.

In addition to autopsy examinations, Galaburda and colleagues $33,40,41$ also performed series of experiments such as inducing cortical anomalies using animal models.

\section{Animal models}

Animal research addresses the question of how minor cortical malformations could lead to clinically persistent disorders of cognitive function ${ }^{33}, 40$. As the small size of ectopias precluded the use of imaging techniques, Galaburda and colleagues studied ectopias that had relatively similar morphology to those present in the human dyslexics using animal models ${ }^{33.41}$. The induction of cortical malformations similar to those found in the dyslexic brain in the rat produced a vari- 
ety of cognitive deficits 33,41 .

\section{2) Structural imaging studies}

The advent of structural imaging techniques (SIT) have helped to address some limitations with the autopsy studies. With the SIT (such as the MRI and CT), measurements of brain structures are performed on living persons.

The autopsy findings of symmetrical PT on dyslexic persons by Galaburda et al ${ }^{21,23-24}$ and the study by Geschwind and Levisky ${ }^{4}$ provided the impetus for neuro-imaging studies of developmental disorders ${ }^{42}$. However, the results from MRI studies ${ }^{43-54}$ were inconsistent. Some MRI studies ${ }^{43-46}$ replicated the findings of symmetrical PT from autopsy studies whereas others did not find symmetrical PT in dyslexic subjects ${ }^{47-54}$. In this paper, only the structural imaging studies conducted using the MRI studies will be reviewed as the MRI is an improved technique over the computed tomography (CT), has higher resolution and therefore gives a much improved definition of neuro-anatomical structures ${ }^{32,55}$. In addition, the studies using the CT did not examine the PT specifically.

Four studies ${ }^{43-46}$ found some symmetry of the PT in the dyslexic subjects. The studies include the report by Larsen et $a l^{43}$ in 1990 , which examined the size and symmetry of the PT in group of 19 dyslexic (mean age $15.1 \pm 0.3$ years) and 19 matched non-dyslexic schoolchildren (mean age, $15.4 \pm 0.4$ years). The gender ratio was the same in both groups (15 males and four females) and the study was conducted in Norway. Participants were matched for age, intelligence, social-cultural factors, and educational environment. Symmetrical PTs were more common in the dyslexic group (70\%) compared with $30 \%$ of the control group. The symmetrical PT were attributed to a larger right PT, based on comparing mean lengths of the PT for children with and without symmetrical PT. Furthermore, the authors ${ }^{43}$ noted that all subjects with pure phonological deficits in reading had symmetrical PT, which was an indication of neuroanatomical basis for phonological processing impairment in dyslexia. In 1993, a study by Kusch et $a^{44}$ in the USA measured the superior surface of the temporal lobe (SSTL) on MRI scans in a sample of 17 dyslexics (Nine males and eight females, mean age, $26.2 \pm 15.0$ years) and 21 control subjects (Eight males, 13 females, mean age, $33.4 \pm 15$ years). The SSTL area was divided into anterior and posterior parts (namely, most of the PT and part of the Heschl's gyrus). The authors found that both the anterior and posterior halves of the SSTL area showed significant leftward asymmetry in non-dyslexics, but showed symmetry in dyslexics. According to the authors, this suggests that among dyslexics, the direction of SSTL asymmetry may serve as a risk factor for the severity of reading comprehension problems. Also from the USA, Duara et al45 in 1991, measured the areas of six bilateral brain segments in the right and left hemispheres, on a horizontal brain section of 21 dyslexics, (twelve males and nine females, mean age, $39 \pm 11$ years) and 29 non-dyslexic subjects (fifteen males and 14 females mean age, $35.3 \pm 10$ years) all right-handed. The authors stated that "The region of the brain that includes most of the planum temporale was found to be symmetrical in our study" and then to the contrary stated that "Dyslexic subjects exhibited asymmetry, with the right side greater than the left side". Consequently, this seeming discrepancy has created some inconsistencies in the reporting of the findings by Duara et $a l^{45}$, with some authors reporting symmetry ${ }^{14-15}$ and others reporting no difference in asymmetry 20,55 . The study by Hynd et al ${ }^{46}$ conducted in the USA in 1990 examined the specificity of deviations in patterns of normal brain asymmetry on MRI scans of 10 dyslexics (eight males and two females, mean age, $9.9 \pm 2.04$ years), 10 with attention deficit disorder/hyperactivity (ADD/H), and 10 (eight males and two females (mean age $11.8 \pm 22.0$ years) ageand sex-matched control children. Some of the dyslexic participants had attention deficit disorder. The authors ${ }^{46}$ concluded that the significant increase in the incidence of PT symmetry seems unique to dyslexia and may be related to deviations in normal patterns of corticogenesis. These findings by Hynd et a ${ }^{46}$ correlates with the postmortem findings that $65 \%$ of normal adult brains are larger on the left side in the region of the PT reported by Geschwind and Levisky ${ }^{4}$. However, their finding of a significantly smaller than normal left PT in the dyslexic differs from the bilaterally large PT reported at autopsy in dyslexics subjects by Galaburda et $a^{23}$. This finding is important because it indicates that abnormal patterns of symmetry/asymmetry do not occur in all clinical groups of children 
but may be relatively specific to dyslexia ${ }^{46}$.

Although the above reviewed studies ${ }^{43-46}$ all found some symmetry of the PT, the methodological approach across these studies varied. There were differences in slice thickness, imaging plane and the definition of anatomical areas measured as well as differences in subjects characteristics. More importantly, there are also variations in the pattern of symmetry across the studies that reported some symmetry among the dyslexic populations, which therefore precludes a uniform comparison across studies.

The studies that did not find symmetry in the PT among the dyslexic subjects include the study conducted in the USA in 1997 by Rumsey et al ${ }^{47}$, which examined the size and asymmetry of the PT and its extension into the parietal lobe (planum parietale) in the brains of 16 right-handed dyslexic men (all males, mean age, $27 \pm 8.0$ years) and 14 matched control subjects all males, mean age $24 \pm 5.0$ years) using the MRI. Approximately $70 \%$ to $80 \%$ of both groups showed equivalent leftward (left $>$ right) asymmetries of the PT. The authors concluded that given the heterogeneity of the dyslexic population, some subgroup of dyslexic individuals might show unusual symmetry in the PT. From Norway in 1999, Heiervang et $a l^{48}$ in a population-based study measured the PT and the adjacent planum parietale (PP) region in sagittal MR images of 20 right-handed dyslexic subjects (mean age, 11.80 years) and 20 matched control subjects (mean age 11.75 years). All the participants in this study were males. The results showed a mean leftward PT asymmetry in both the dyslexic and the control group, with no significant difference for the degree of PT asymmetry. Also from Norway and from the same group of researchers Hugdahl et al ${ }^{49}$ investigated differences between dyslexic and normal reading children in asymmetry of the PT area in the upper posterior part of the temporal lobe. The participants were 23 dyslexic (nineteen males and four females, the age range indicated was $10-12$ years) and 23 normal reading children (twenty males and three females, age range indicated was 10-12 years). Hand preference was indicated. The authors found a significantly larger left than right PT area for both groups. The authors noted that while the right PT area was similar for the dyslexic and control groups, the left PT was significantly smaller in the dyslexic group. In the 1994 detailed study conducted by Schultz et $a l^{50}$ in the USA that controlled for gender, age and handedness, MRI techniques were used to compare the convolution surface area of the PT, temporal lobe volume and superior surface area of 17 dyslexic children (ten males and seven females, mean age $8.68 \pm$ 0.64 years) and 14 non-dyslexic children (seven males and seven females, mean age $8.94 \pm 0.67$ years). All subjects were right-handed. According to the authors, the initial analyses suggested smaller left hemisphere structures in dyslexics compared to control subjects whereas subsequent analyses controlling for age, gender and overall brain size revealed no significant differences between dyslexics and non-impaired children on a variety of measures, in particular surface area and symmetry of the PT. Leonardo et a ${ }^{51}$ studied anatomical structure (including the PT) in 15 reading disabled (RD) subjects (eight males, seven females, mean age $24 \pm 3.0$ years) and 15 control (eight males and 7 females, mean age $22 \pm 3.0$ years) in a volumetric MRI scan. This study was conducted in the USA. The RD group had a more marked leftward asymmetry of the PT, although the group difference was non-significant ${ }^{51}$. Another study conducted by Leonardo et al in 1993 (cited in Leonardo et al) ${ }^{51}$ also found a leftward asymmetry of the PT. Robicho et al ${ }^{52}$ in 1999 measured cortical asymmetries of posterior language-related areas, including the PT in 16 adult male dyslexic subjects (mean age $21 \pm 0.2$ years) and 14 age-matched male controls (mean age $23.6 \pm 3.9$ years) in France. Nine of the 16 dyslexic subjects were right-handed and four of the controls were not. They found no differences in PT asymmetry between the two groups and concluded that phonological segmentation skills may relate to frontal lobe morphology, while phonological memory-based impairment in people with dyslexia may rather relate to parietal lobe asymmetry. Using similar methods as in the study by Robichon et $a l^{52}$ another study by the same group of researchers, Habib and Robichon ${ }^{53}$ also found no differences in PT asymmetry. The study by Best and Demb $^{54}$ presented another perspective. The authors measured the PT in five dyslexic subjects (three males and two females, mean age $22 \pm 2.9$ years) with a documented magnocellular deficit and five controls subjects (Three males, two females mean age $26.8 \pm$ 6.1 years). The study was reported in 1999 and all the dyslexic subjects showed normal, leftward asymmetry of the PT ${ }^{54}$. Best and Demb ${ }^{54}$ concluded that 
planar symmetry may be associated with a subgroup of dyslexia.

The reviewed studies ${ }^{47-54}$ that did not find symmetrical PT in dyslexic population also has methodological variations across the studies. Despite a similar report of non-symmetrical PT, the findings of these studies differed in some ways, for example, Heiering et $a l^{48}$ and Hughal et a ${ }^{49}$ found leftward PT asymmetry in both the dyslexic and the control group with the dyslexics having smaller left PT. Schutz et al ${ }^{50}$ found no significant differences between dyslexics and nondyslexic on a subsequent analysis while Leonardo et $a l^{51}$ found exaggerated leftward asymmetry of the PT in the dyslexic group.

Taken together, a trend with autopsy and MRI studies on the dyslexic population is that results appeared to be consistent across groups of researchers. For example, Galaburda and colleagues ${ }^{21-22}$ consistently found symmetrical PT in all their postmortem subjects. Leonarndo et al ${ }^{51}$ study found exaggerated leftward asymmetry. Robicho et al ${ }^{52}$ and Habib and Robichon ${ }^{53}$ reported no differences in PT asymmetry between dyslexics and non-dyslexics. This tendency may be an indication of some methodological bias.

\section{Discussion}

Autopsy studies on dyslexia have been conducted by the same group of researchers (Galaburda et al) and the results are fairly consistent with all subjects examined having symmetrical PT and several with cortical malformation, mainly ectopias and dyspla$\operatorname{sia}^{21,23-24}$. However, results from the MRI studies reviewed on $\mathrm{PT}^{43-54}$ have shown an inconsistent trend, for example, the autopsy findings of Galaburda and colleagues have been replicated only in a few of the MRI studies ${ }^{43-46}$. The inconsistencies in the findings reported by different researchers have been attributed to methodological differences across studies ${ }^{14,50}$. The limitations may be broadly classified as: issues related to measurements and technique (definition of anatomical borders, dimensions measured, slice thickness, measurement plane) as well as subjects' characteristics (gender, age, handedness, sample size and differences in dyslexia diagnostic standards, and co-diagnoses) as well as intelligence quotient. These limitations are discussed below.

Specifically, a major difficulty in studies conduct- ed on PT is related to the definition of its anterior and posterior borders ${ }^{14,55,56}$. Studies ${ }^{14,56,57}$ have shown that the way the anatomical borders of the PT is defined affects the measurement outcome. Zetzche et $a l^{56}$ have outlined different methods of description of the PT and remarkably, studies on PT have used different definitions of the PT. In particular, there has been confusion as to exactly what the PT is and whether the second Heschl's gyrus, (if present) should be included and which one of the planes created by the posterior branching of the Sylvian fissures constitutes the continuation of the PT ${ }^{58}$. Therefore, issues related to the inconsistencies in the definition of the PT has to be resolved before results by the different researchers can be appropriately compared $14,56,58$. The difficulty of determining the exact anatomical definition of the PT also led to some authors measuring some indirect structures, as in the study by Kusch et $a^{44}$. Also, inter-measurer reliability in the measurements of the PT is poor ${ }^{55}$.

Another possible source of inconsistent results in PT research relates to the dimension of PT measurements. Invariably, the size of structures of the brain can be estimated using linear, area, or volumetric measurements ${ }^{14}$ and different studies have used different dimensions. The use of any measurement dimension, which does not account for the convoluted nature of the PT, is likely to obtain unreliable results ${ }^{14,60}$.

Furthermore, different MR imaging plane (axial/ horizontal, coronal or sagittal) have been used in different studies to measure shape, size and asymmetry of the PT. According to Galaburda ${ }^{58}$ although each imaging plane has unique advantages, there are also limitations with individual technique. For example, a demerit with the commonly employed horizontal (axial) plane in MRI is that it is too close to the plane of the PT itself, making visualization difficult even for experienced investigators ${ }^{14,58}$. Related to the problem of imaging plane is that different MRI slice thicknesses (Hynd et al ${ }^{46}=7.5 \mathrm{~mm}$, Larsen et al ${ }^{43}=3 \mathrm{~mm}$, Duara et al ${ }^{45}=7.00 \mathrm{~mm}$, Kusch et al ${ }^{46}=5 \mathrm{~mm}$, Schultz et $a l^{50}=5 \mathrm{~mm}$, Leonardo and Eckert ${ }^{51}=1 \mathrm{~mm}$ ) have been employed in different studies.

One of the factors related to subjects' characteristics is gender. Some MRI studies combined the results from disproportionate ratios of male and female subjects and controls despite evidence that gender is an important variable in brain morphology. Support for 
the hypothesis that brain language areas may be differently organized in males and females comes from autopsy studies ${ }^{24}$, MRI studies ${ }^{17,61-62}$ and a functional magnetic imaging (fMRI) study by Shaywitz et al63 who demonstrated gender differences in the localization of brain activation during phonological processing. In addition, girls and boys differ in language development ability ${ }^{64}$ and that reading disability affect boys more frequently than girls, with reported gender ratios ${ }^{65-66}$ of $3: 1$. Also, on average, men have $8-10 \%$ larger brains than women, therefore the size of a particular brain structure, such as the PT, can also be expected to be larger in men ${ }^{16}$. Examples of studies with disproportionate gender ratio are the study by Heivering et al ${ }^{48}$, where all participants from both groups were males and the study by Hugdahl et al ${ }^{49}$ where subjects from the dyslexic group consisted of 19 boys and four girls and 20 boys and three girls for the control group.

Another methodological variable related to subject characteristics is handedness (dominant hand/hand preference). Determining the subjects' hand preferences is important because handedness is believed to relate to functional language lateralization and individuals with consistent right-handedness have been reported to show more pronounced asymmetries of the PT ${ }^{14,16,47,56,67-68}$. Strict controls for handedness are essential in anatomical studies as failure to do so is a major methodological flaw ${ }^{32}$.

Finally, other methodological factors, which may contribute to differences in findings across studies, include age, small sample sizes, criteria used to define dyslexia, the heterogeneity of the disorder, and co-diagnoses (such as attention-deficit/hyperactivity disorder, specific language impairment). Age as a variable may constitute a confounder due to the effects of brain development which relates to a possible difference between child and adult studies ${ }^{14}$. The samples sizes across studies are typically small with an average of 15 for dyslexic subjects for the studies reviewed. Small sample sizes do not have a high statistical power to detect differences in variables such as handedness, age and gender differences. For example, the study by Schultz et al ${ }^{50}$ demonstrated a significant influence of gender and age on brain morphology. In addition, diagnostic criteria for dyslexia varied across studies ${ }^{43-54}$.

\section{Recommendations for future studies}

Given the outlined limitations with the studies reviewed, different authorities have recommended various methodological modifications aimed to improve the consistencies from neuro-imaging studies. These recommendations are summarized below.

1) A clear operational definition of the anatomical borders of the PT must be established and used across studies.

2) Slice thickness ranging between $1.5-2.0 \mathrm{~mm}$ which will allow good visualization of cortical foldings and enables accurate identification of the PT boundaries is recommended ${ }^{14,32}$.

3) Coronal rather than sagittal imaging sequences are preferred because these yield more distinct image slices through the PT. Also since the PT is a convoluted three-dimensional structure, no single plane is optimal for display of the PT. Only the volumetric MRI acquisitions allows the use of a computer programme that allows simultaneous display in all three orthogonal views, in the axial, sagittal and coronal planes which substantially improves anatomical border determination and has been suggested by some studies ${ }^{14,16,58}$.

4) Sample sizes must be large enough to allow a high statistical power to adequately address variables such as handedness, age and gender differences ${ }^{69}$.

5) Handedness, age and gender difference should be adequately controlled. Accurate measurement, preferably objective and subjective of handedness is suggested ${ }^{14}$.

The possible limitation with this review is that the literature search is limited to English-only sources. However, the major strength is that it is a thorough and up-to-date review paper on the neuro-anatomy of developmental dyslexia.

\section{Summary and Conclusion}

Overall, only the autopsy investigations conducted by Galaburd et al21,23-24 have been consistent in their findings of unusual symmetry of the PT, cortical malformations and differences in the thalamus. The reports from MRI studies were inconsistent possibly due to methodological variations across the studies. 
Although the occurrence of symmetrical PT may not confirm the development of dyslexia, the occurrence of symmetrical PT in several consecutively autopsied subjects may not simply be due to chance. Also, given that these same findings (PT symmetry) have been corroborated by some MRI studies suggests that symmetrical PT may be relevant in the etiology of DD. However, the fact that there are also variations in the pattern of symmetry reported across the studies that found some symmetry of the PT (in MRI studies) among the dyslexic populations makes it difficult to make a firm conclusion in support of symmetrical PT in dyslexia. Therefore, given the available evidence from the reviewed studies, no anatomical structure appears to firmly characterize dyslexic individuals.

The neurobiology of dyslexia remains a subject of continued research and it will be important to investigate the relevance of other neuro-anatomical structures in the etiology of dyslexia. Consequently, Galaburda ${ }^{25}$ reported that the presence of enlarged PT associated with symmetrical PT may be related to increased number of axons passing through the corpus callosum (nerve fibre connecting both hemispheres of thee brain) which then creates an anomalous interhemispheric pathways to the perisylvian-language regions. Due to its role in interhemispheric transfer, the corpus callosum has been another area of intense research in the quest for the neurobiology of DD. Evidence from MRI studies of the CC will be reviewed in part 2 of the series on the neurobiology of development dyslexia. This review is intended to contribute to the understanding of dyslexia and to guide future research.

\section{Acknowledgement}

I am grateful to Professor Kovin Naidoo and Percy Mashige for reviewing the final draft of the manuscript.

\section{References}

1. Krupska M, Klein C. Demystifying Dyslexia. London Language and Literacy Unit, 1995.

2. Habib M. The neurological basis of developmental dyslexia. An overview and working hypothesis. Brain $2000 \mathbf{1 2 3}$ 2373-2399.

3. Nakada, T Fujii Y. Yoneoka Y. Kwee IL. Planum temporale: Where spoken and written language meet. Eur Neurol 2001 46 121-125.

4. Geschwind N, Levitsky W. Human brain: Left-right asymmetries in the temporal speech region. Science $1968 \mathbf{1 6 1}$ 186-187.
5. Leonard CM. Imaging brain structure in children: Differentiating language disability and reading disability. Learn Disab Quart 200124 158-176.

6. Shaywitz S, Shaywitzx BA. The science of reading and dyslexia. J Am Assoc Ped Ophthalmol Strab 20037 158-166.

7. Lambe EK. Dyslexia, gender and brain imaging. Neuropsychologia 199937 521-537.

8. Rae C, Harasty, JA, Dzendrowskyj TE, Talcott JB, Simpson JM, Blamire AM, Dixon, RM. Cerebellar morphology in developmental dyslexia. Neuropsychologia 2002401285 1292.

9. Galaburda AM. Dyslexia: A molecular disorder of neuronal migration. Annals Dyslexia 200555 151-165.

10. Semrud-Clikeman M. Evidence from imaging on the relationship between brain structure and developmental language disorders. Sem in Ped Neur 19974 (2) 117-124.

11. Zeffiro T, Eden.G. The neural basis of developmental dyslexia. Annals Dyslexia 200050 1-30.

12. Bernstein D, Alison CS, Roy E, Srull T. Psychology. Boston: Houghton Mifflin Publishers, 1994 pg 93-117.

13. http://www.ruf.rice.edu/ lngbrain/cglidden/temporal.html Language and brain. Rice University lecture notes Date Accessed 27th March 2011.

14. Shapleske J, Rossell SL, Woodruff PW, David AS. The planum temporale: a systematic, quantitative review of its structural, functional and clinical significance $\mathrm{Br}$ Res Revs 199929 26-49.

15. Leonard CM, Eckert MA. Asymmetry and dyslexia. Develop Neuropsych 200833 (6) 663-681.

16. Dos Santos Sequeira, Woerner W, Walter C, Kreuder F, Lueken U, Westerhausen R Handedness, dichotic-listening ear advantage, and gender effects on planum temporale asymmetry. A volumetric investigation using structural magnetic resonance imaging Neuropsychol 200644 622-636.

17. Kulynych JJ, Vladar K, Jones DW, Weinberger DR. Gender differences in the normal lateralization of the supratemporal cortex: MRI Surface-rendering morphometry of Heschl's Gyrus and the planum temporale. Cerebral Cortex 19944 107-118.

18. Foundas AL. Anatomical basis of language. Top Lang Disord 200121 (3) 1-19.

19. Vellutino FR, Fletcher JM. Snowling MJ. Scanlon DM. Specific reading disability (dyslexia): what have we learned in the past four decades? J Child Psycho Psych 200445 2-40.

20. Morgan AE, Hynd GW. Dyslexia, neurolinguistic ability, and anatomical variation of the planum temporale. $\mathrm{Neu}$ ropsych Rev1998 8 (2) 79-93.

21. Galaburda AM, Kemper TL. Cytoarchitectonic abnormalities in developmental dyslexia: A case study. Annals Neurol 19796 94-100.

22. Binder JR, Frost JA, Hammeke TA, Rao SM, Cox W. Function of the left planum temporale in auditory and linguistic processing. Brain 1996119 1239-1247.

23. Galaburda AM, Sherman GE, Rosen GD, Aboitiz F, Geschwind N. Developmental dyslexia: Four consecutive patients with cortical anomalies. Annals Neurol 198518222 233.

24. Humphreys P, Kaufmann W, Galaburda AM. Developmental dyslexia in women: Neuropathological findings in three patients. Annals Neurol 199028 727-738. 
25. Galaburda A M. Ordinary and extraordinary brain development. Anatomical variation in developmental dyslexia. $A n-$ nals Dyslexia 198939 67-80.

26. Galaburda, AM, Sanides F. Cytoarchitectonic organization of the human auditory cortex. J Comp Neurol 1980190597 610 .

27. Jenner AR, Rosen GD, Galaburda AM. Neuronal asymmetries in primary visual cortex of dyslexic and non-dyslexic brains. Annals Neurol 199946 (2)189-196.

28. Galaburda AM, Menard MT, Rosen GD. Evidence for aberrant auditory anatomy in developmental dyslexia. Proc Nat Acad Sci 199491 8010-8013.

29. Livingstone M, Rosen G, Drislane F, Galaburda AM. Physiological and anatomical evidence for a magnocellular defect in developmental dyslexia. Proc Nat Acad Sc 1991887943 7947.

30. Drake W. Clinical and pathological findings in a child with a developmental learning disability. J Learn Disabil 19681 (9) 486-502.

31. Galaburda AM, Corsiglia J. Rosen GD, Sherman GF. Planum temporale asymmetry: Reappraisal since Geschwind and Levitsky Neuropsych 198725 (6) 853-868.

32. Rumsey J. Developmental dyslexia: Anatomical and functional neuroimaging. Ment Retard Develop Dis Res Rev $1996228-36$

33. Galabuda AM. Brains and Sounds. Lessons from "Dyslexic" rodents. The MIT Press 2001 448-461.

34. Galaburda AM. Developmental dyslexia: Multilevel syndrome. Dyslexia 19995 183-191.

35. Galaburda AM, LoTurco J, Ramus F, Fitch RH, Rosen GD. From genes to behaviour in developmental dyslexia. Nature Neurosc 20069 (10) 1213-1217.

36. http://sintn-seminars.stanford.edu/reprints/Galaburda1. LoTurco J, Yu Wang Paramasivam, M. Neuronal migration and dyslexia susceptibility. Last Acessed 3rd April 2011.

37. Heim S, Keil A. Large-scale neural correlates of developmental dyslexia. Europ Child \& Adoles Psychiat 200413 (3) 124-140.

38. Beaton, AA. The relation of planum temporale asymmetry and morphology of the corpus callosum to handedness, gender, and dyslexia: a review of the evidence. Brain Lang 199760 255-322.

39. Hynd GW, Semrud-Clikeman, M. Dyslexia and neurodevelopmental pathology: Relationships to cognition, intelligence, and reading skill acquisation. J Learn Dis 1989 22 (4) 204-220.

40. Galaburda AM. Developmental dyslexia and animal studies: at the interface between cognition and neurology. Cognition 199450 133-149.

41. Herman AE, Galaburda AM, Fitch HR, Carter AR, Rosen GD. Cerebral microgyria, thalamic cell size and auditory temporal processing in male and female rats. Cerebral Cortex 19977 453-464.

42. Watkins KE, Gadian DG, Vargha-Khadem F. Functional and structural brain abnormalities associated with a genetic disorder of speech and language. Am J Hum Genet 199965 1215-1221.

43. Larsen JP, Høien T, Lundberg I, Ødegaard H. MRI evaluation of the size and symmetry of the planum temporale in adolescents with developmental dyslexia. Brain Lang 1990 39 289-301.

44. Kushch A,Gross-Glenn K, Jallad B, Lubs H, Rabin M, Feldman E, Duara R. Temporal lobe surface area measurements on MRI in normal and dyslexic readers. Neuropsychol 1993 31 811-821.

45. Duara R, Kushch A, Gross-Glenn K, Barker WW, Jallad B, Pascal S, Loewenstein DA, Sheldon J, Rabin M, Levin B, Lubs H. Neuroanatomic differences between dyslexic and normal readers on magnetic resonance imaging scans. Arch Neurol 199148 410-416.

46. Hynd GW, Semrud-Clikeman M, Lorys AR, Novey ES, Eliopulos D. Brain morphology in developmental dyslexia and attention deficit disorder/hyperactivity. Arch Neurol 199047 919-926.

47 Rumsey JM, Donohue BC, Brady DR, Nace K, Giedd JN, Andreason P. A magnetic resonance imaging study of planum temporale asymmetry in men with developmental dyslexia. Arch Neurol 199754 481-1489.

48. Heiervang E, Hugdahl K, Steinmetz H, Smievoll AI, Stevenson J, Lund A, Ersland L, Lundervold A. Planum temporale, planum parietale and dichotic listening in dyslexia. Neuropsychol 200038 1704-1713.

49. Hugdahl K, Heiervang E, Ersland L, Lundervold A, Steinmetz H, Smievoll A. Significant relation between MR measures of planum temporale area and dichotic processing of syllables in dyslexic children. Neuropsych 412003 666-675.

50. Schultz RT, Cho NK, Staib LH,Kier LE, Fletcher JM, Shaywitz SE, Shankweiler DP, Katz L, Gore JC, Duncan JS, Shaywitz BA. Brain morphology in normal and dyslexic children: The influence of sex and age. Annals Neurol 1994 35 732-742.

51. Leonard CM, Eckert MA, Lombaedino LJ, OAKland T, Kranzler J, Mohr C. Anatomical risk factors for phonological dyslexia. Cerebral Cortex 200111 148-157.

52. Robichon F, Levrier O, Farnarier P, Habib M. Developmental dyslexia: Atypical cortical asymmetries and functional significance. Europ J Neurol 20007 35-46.

53. Habib M, Robichon F. Parietal lobe morphology predicts phonological skills in developmental dyslexia. Brain Cogn 199632 139-142.

54. Best M, Demb JB. Normal planum temporale asymmetry in dyslexics with a magnocellular pathway deficit. Neurorep 199910 607-612.

55. Dalby MA, Elbro C, Stødkilde-Jørgensen H. Temporal lobe asymmetry and dyslexia: An in vivo study using MRI. Brain Lang 199862 51-69.

56. Zetzsche T, Meisenzahla EM, Preussa UW, Holdera JJ, Kathmanna N, Leinsinger G, In-vivo analysis of the human planum temporale: does the definition of PT borders influence the results with regard to cerebral asymmetry and correlation with handedness? Psy Res Neuroimaging Section 2001 107 99-115.

57. Tervaniemi M, Hugdahl K. Lateralization of auditory-cortex functions. Brain Res Revs 200343 231-246.

58. Galaburda AM. The Planum temporale. Arch Neur 1993 50457.

59. Habib M, Robichon F, Levrier O, Khalil R, Salamon G. Diverging Asymmetries of Temporo-parietal Cortical Areas: 
60. Barta P E, Petty RG, McGilchrist I, Lewis RW, Jerram M, Casanova M, Powers R. Asymmetry of the planum temporale: Methodological considerations and clinical associations. Neuroimaging 199561 137-150.

61. Good CD, Johnsrude I, Ashburner J, Henson RN, Friston KJ, Frackowiak RS. Cerebral asymmetry and the effects of sex and handedness on brain structure: A voxel-based morphometric analysis of 465 normal adult human brains. NeuroImage $200114685-700$.

62. Knaus TA, Bollich AM, Corey DM, Lemen LC, Foundas AL. Sex-linked differences in the anatomy of the perisylvian language cortex: A volumetric MRI study of gray matter volumes. Neuropsych 200418 4738-4747.

63. Shaywitz BA, Pugh KR, Fulbright RK. Functional disruption in the organization of the brain for reading in dyslexia. Proc Nat Acad Sci 199895 2636-2641.

64. Sommer IE, Aleman A, Somers M, Boks MP, Kahn RS. Sex differences in handedness, asymmetry of the planum temporale and functional language lateralization. Brain Res 2008 1206 76-88.

65. Wajuihian SO, Naidoo KS. Dyslexia. An Overview. $S$ Afr Optom 201170 (2) 89-98.

66. Liederman J, Kantrowitz L, Flannery K. Male vulnerability to reading disability is not likely to be a myth: a call for new data. J Learn Disabil 200538 (2) 109-129.

67. Hagmann P, Cammoun L, Martuzzi R, Maeder P, Clarke S, Thiran J Meul R. Hand preference and sex shape the architecture of language networks. Human Brain Mapping 2006 27 828-835.

68. Foundas AL, Leonard CM, Hanna-Pladdy B. Variability in the anatomy of the planum temporale and posterior ascending ramus: Do right- and left handers differ? Brain Lang 200283 403-424.

69. Eckert MA, Leonardo CM. Structural imaging in dyslexia: The planum temporale Ment Retard Develop Dis Res Rev 20026 198-206. 